F. Chamizo and H. Iwaniec

Nagoya Math. J.

Vol. 151 (1998), 199-208

\title{
ON THE GAUSS MEAN-VALUE FORMULA FOR CLASS NUMBER
}

\author{
FERNANDO CHAMIZO AND HENRYK IWANIEC
}

\begin{abstract}
In his masterwork Disquisitiones Arithmeticae, Gauss stated an approximate formula for the average of the class number for negative discriminants. In this paper we improve the known estimates for the error term in Gauss approximate formula. Namely, our result can be written as $N^{-1} \sum_{n \leq N} H(-n)$ $=4 \pi \sqrt{N} /(21 \zeta(3))-2 \pi^{2}+O\left(N^{-15 / 44+\epsilon}\right)$ for every $\epsilon>0$, where $H(-n)$ is, in modern notation, $h(-4 n)$. We also consider the average of $h(-n)$ itself obtaining the same type of result.

Proving this formula we transform firstly the problem in a lattice point problem (as probably Gauss did) and we use a functional equation due to Shintani and Dirichlet class number formula to express the error term as a sum of character and exponential sums that can be estimated with techniques introduced in a previous work on the sphere problem.
\end{abstract}

\section{$\S 1$. Introduction and statement of the main result}

Gauss noted in Art. 302 of [Ga] that the average of the class number for negative discriminants increases very regularly, and he gave (without proof) an approximate formula for this average, checking its accuracy up to unbelievably large values. (He, in fact, computed not only the class number but also the subdivision into genera for several thousands of discriminants).

Probably Gauss proved that his formula gives the correct asymptotics but the first known proof of this fact is due to Lipschitz in 1865. In this century I. M. Vinogradov studied the error term in Gauss' approximation using the method of trigonometric sums. In a series of papers, along fifty years, he gave several upper bounds that also hold for the "sphere problem" (the three-dimensional analogue of the circle problem).

In $[\mathrm{Ch}-\mathrm{Iw}]$ we improved the results of Chen [Ch] and Vinogradov [Vi] on the sphere problem. The key point in our method is the double interpretation of the sphere problem; the first one leads us to trigonometric sums and the second one to character sums. The purpose of this paper is to apply

Received August 5, 1996. 
the same method to Gauss' original problem to get a similar upper bound for the error term.

Before stating our main results we shall mention some notions of classical and modern theory of positive definite binary quadratic forms in order to introduce some notation and facilitate posterior references:

For each positive integer, $n$, we consider the set $\mathcal{Q}_{n}$ of positive definite quadratic forms of discriminant $-n$

$$
\mathcal{Q}_{n}=\left\{Q(x, y)=a x^{2}+b x y+c y^{2}: 4 a c-b^{2}=n \text { with } a>0\right\} .
$$

We also define the set of primitive forms

$$
\mathcal{Q}_{n}^{*}=\left\{Q \in \mathcal{Q}_{n}: \operatorname{gcd}(a, b, c)=1\right\}
$$

and the set of primitive forms in the sense of Gauss

$$
\mathcal{Q}_{n}^{\prime}=\left\{Q \in \mathcal{Q}_{4 n}^{*}: 2 \mid b\right\}
$$

The modular group $\Gamma=S L_{2}(\mathbb{Z})$ acts on $\mathcal{Q}_{n}, \mathcal{Q}_{n}^{*}$ and $\mathcal{Q}_{n}^{\prime}$ by the rule

$$
Q^{\gamma}(x, y)=(x, y) \gamma^{t}\left(\begin{array}{cc}
a & b / 2 \\
b / 2 & c
\end{array}\right) \gamma\left(\begin{array}{l}
x \\
y
\end{array}\right), \quad \gamma \in S L_{2}(\mathbb{Z}) .
$$

When $n \equiv 0,3(4)$, the set of primitive quadratic forms, $\mathcal{Q}_{n}^{*}$, is non-empty and following modern authors, its class number, say $h(-n)$, is defined as the number of orbits when $\Gamma$ acts on $\mathcal{Q}_{n}^{*}$. Similarly, the class number of all positive definite quadratic forms of discriminant $-n$, say $N(n)$, is defined to be the number of orbits when $\Gamma$ acts on $\mathcal{Q}_{n}$. Clearly

$$
N(n)=\sum_{k^{2} \mid n} h\left(-n / k^{2}\right)
$$

Gauss defined the class number of primitive quadratic forms, say $H(-n)$, considering $\mathcal{Q}_{n}^{\prime}$ instead of $\mathcal{Q}_{n}^{*}$. This definition is natural if we want the matrix of each quadratic form to have integral entries, but the relation with the theory of ideals in quadratic fields supports the modern definition.

The class number can be computed by the formula (see [Da])

$$
\begin{array}{r}
h(-n)=\frac{w_{-n} \sqrt{n}}{2 \pi} L\left(1, \chi_{-n}\right), \quad \text { where } \chi_{d}(m)=\left(\frac{d}{m}\right) \\
\text { and } w_{d}= \begin{cases}2 & \text { if } d<-4 \\
4 & \text { if } d=-4 \\
6 & \text { if } d=-3 .\end{cases}
\end{array}
$$


Comparing this expression with the classical formula proved by Dirichlet for Gauss class number (see $\S 97$ of [Di]) it follows $H(-n)=h(-4 n)$.

After these considerations, we can state our main results:

THEOREM 1.1. For $N \geq 1$ and any $\epsilon>0$

$$
\sum_{n \leq N} H(-n)=\frac{4 \pi}{21 \zeta(3)} N^{3 / 2}-\frac{2}{\pi^{2}} N+O\left(N^{29 / 44+\epsilon}\right) .
$$

TheOrem 1.2. For $N \geq 1$ and any $\epsilon>0$

$$
\sum_{n \leq N} h(-n)=\frac{\pi}{18 \zeta(3)} N^{3 / 2}-\frac{3}{2 \pi^{2}} N+O\left(N^{29 / 44+\epsilon}\right) .
$$

\section{$\S 2$. Class number and lattice points}

Trying to keep the analogy with the notation of [Ch-Iw], we define:

$$
\mathcal{H}_{1}(R)=\sum_{n \leq R^{2}} H(-n) \quad \text { and } \quad \mathcal{H}_{2}(R)=\sum_{n \leq R^{2}} h(-n)
$$

In this section we shall write this quantities in terms of the number of lattice points in certains regions, the basic result is the following lemma, which is a reformulation in modern notation of Art. 172 and 174 of [Ga]:

\section{Lemma 2.1. For $n \geq 1$}

$$
N(n)=\#\left\{(a, b, c): 4 a c-b^{2}=n,-a<b \leq a<c \text { or } 0 \leq b \leq a=c\right\} .
$$

Proof. If we assign to each quadratic form $a x^{2}+b x y+c y^{2}$ in $\mathcal{Q}_{n}$ the complex number $w=(-b+i \sqrt{n}) / 2 a$ in the upper half-plane, $\mathbb{H}$, then the action of the modular group on these points is in one-to-one correspondence with the action of $\Gamma /\{ \pm I\}$ on $\mathcal{Q}_{n}$. Hence $N(n)$ is the cardinality of the complex numbers $w=(-b+i \sqrt{n}) / 2 a$ with $4 a c-b^{2}=n$ belonging to the standard fundamental domain of $\Gamma /\{ \pm I\}$

$\mathcal{F}=\{z=x+i y \in \mathbb{H}:|z|>1,-1 / 2<x \leq 1 / 2$ or $|z|=1,0 \leq x \leq 1 / 2\}$

This proves the lemma. 
Next we introduce some smooth weights to the sums $\mathcal{H}_{1}(R)$ and $\mathcal{H}_{2}(R)$.

Given $R>1$ and $\Delta=\Delta(R)>0$, let $f_{R}$ be the function supported in $[0, R+\Delta]$ defined by

$$
f_{R}(x)=\left\{\begin{array}{cl}
x & \text { if } 0 \leq x \leq R \\
R(R+\Delta-x) / \Delta & \text { if } R \leq x \leq R+\Delta .
\end{array}\right.
$$

then the following result holds

LEMma 2.2. For $R>1$ and $\Delta=\Delta(R)>0$, we have

$$
\mathcal{H}_{1}(R)=\sum_{2 \nmid k} \mu(k)\left(\Phi_{1}(R / k)-\Phi_{2}(R / k)+\Psi_{1}(R / k)\right)
$$

and

$$
\mathcal{H}_{2}(R)=\sum_{k} \mu(k)\left(\Phi_{2}(R / k)+\Psi_{2}(R / k)\right)
$$

where

$$
\Phi_{1}(R)=\sum_{n} \frac{N(4 n)}{\sqrt{n}} f_{R}(\sqrt{n}) \quad \Phi_{2}(R)=\sum_{n} \frac{N(n)}{\sqrt{n}} f_{R}(\sqrt{n})
$$

and

$$
\begin{aligned}
& \Psi_{1}(R)=-\sum_{R<l \sqrt{n} \leq R+\Delta(R)} \sum_{\substack{2 \nmid l \\
\text { (n) }}} H(-n) \frac{f_{R}(l \sqrt{n})}{l \sqrt{n}}
\end{aligned}
$$

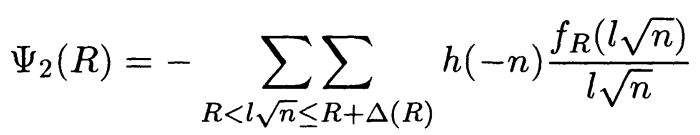

Proof. We shall prove the lemma only for $\mathcal{H}_{1}(R)$, the proof for $\mathcal{H}_{2}(R)$ is similar. We start with the formula

$$
\mathcal{H}_{1}(R)=\sum_{n \leq R^{2}} h(-4 n)=\sum_{n \leq R^{2}} \sum_{k^{2} \mid 4 n} \mu(k) N\left(4 n / k^{2}\right),
$$

interchanging the order of summation

$$
\begin{aligned}
\mathcal{H}_{1}(R) & =\sum_{k} \sum_{\substack{n \leq 4 R^{2} k^{-2} \\
4 \mid n k^{2}}} \mu(k) N(n) \\
& =\sum_{2 \mid k} \mu(k) \sum_{n<4 R^{2} k^{-2}} N(n)+\sum_{2 \nmid k} \mu(k) \sum_{n<R^{2} k^{-2}} N(4 n),
\end{aligned}
$$


hence

$$
\begin{aligned}
\mathcal{H}_{1}(R) & =\sum_{2 \nmid k} \mu(k) \sum_{n<R^{2} k^{-2}} N(n)+\sum_{2 \nmid k} \mu(k) \sum_{n<R^{2} k^{-2}} N(4 n) \\
& =\sum_{2 \nmid k} \sum_{n<R^{2} k^{-2}} \mu(k)(N(4 n)-N(n)) .
\end{aligned}
$$

Using that $f_{R}(\sqrt{x}) / \sqrt{x}=1$ for $0<x \leq R$, we have

$$
\mathcal{H}_{1}(R)=\sum_{2 \nmid k} \mu(k)\left(\Phi_{1}(R / k)-\Phi_{2}(R / k)-S(R / k)\right)
$$

where

$$
\begin{aligned}
S(R) & =\sum_{R<\sqrt{n}<R+\Delta(R)}(N(4 n)-N(n)) \frac{f_{R}(\sqrt{n})}{\sqrt{n}} \\
& =\sum_{R<\sqrt{n}<R+\Delta(R)} \frac{f_{R}(\sqrt{n})}{\sqrt{n}}\left(\sum_{l^{2} \mid 4 n} h\left(-4 n / l^{2}\right)-\sum_{l^{2} \mid n} h\left(-n / l^{2}\right)\right) \\
& =\sum_{R<\sqrt{n}<R+\Delta(R)} \sum_{\substack{l^{2} \mid n \\
2 \nmid l}} h\left(-4 n / l^{2}\right) \frac{f_{R}(\sqrt{n})}{\sqrt{n}}=-\Psi_{1}(R)
\end{aligned}
$$

and substituting in (2.1) the lemma follows.

\section{$\S 3 . \quad$ A weighted Voronoï formula}

In this section we shall state a transformation formula for weighted sums of $N(n)$ based on the functional equation of a zeta function considered by Shintani, and we shall use it to derive a kind of Voronoï formula for the quantities $\Phi_{1}$ and $\Phi_{2}$ defined in Lemma 2.2.

Let us define

$$
N^{*}(n)=2 \sum_{k^{2} \mid n} h\left(-n / k^{2}\right) w_{-n / k^{2}}^{-1}
$$

Note that $N^{*}(n)$ equals $N(n)$ except when $n=3 k^{2}$ or $n=4 k^{2}$, in these cases $N^{*}(n)-N(n)=-2 / 3$ or $-1 / 2$ respectively.

LEMMA 3.1. Let $g \in C_{0}^{\infty}([0, \infty))$ with $g(0)=g^{\prime \prime}(0)=0$, then

$$
\sum_{n=1}^{\infty} \frac{N^{*}(4 n)}{\sqrt{4 n}} g(\sqrt{4 n})=\frac{1}{12} G^{\prime}(0)-\sum_{n=1}^{\infty} \frac{N^{*}(n)}{\sqrt{n}} \tilde{g}(\sqrt{n})-\frac{1}{2} \int_{0}^{\infty} \frac{G(r)}{\operatorname{sh} \pi r} d r
$$


where

$$
\tilde{g}(x)=\int_{0}^{\infty} g(t) \sin (\pi x t) d t \quad \text { and } \quad G(x)=g(x)+\tilde{g}(x) .
$$

Proof. Defining $f(x)=2 G(x)$, under our hypothesis on $g$, the series

$$
\begin{aligned}
S & =\sum \frac{N^{*}(4 n)}{\sqrt{4 n}} f(\sqrt{4 n})-\frac{1}{2} \sum \frac{N^{*}(n)}{\sqrt{n}} f(\sqrt{n}) \\
S^{\prime} & =\sum \frac{N^{*}(4 n)}{\sqrt{4 n}} \tilde{f}(\sqrt{4 n})-\sum \frac{N^{*}(n)}{\sqrt{n}} \tilde{f}(\sqrt{n}),
\end{aligned}
$$

converge absolutely and the lemma reduces to prove (note that $g=f-\tilde{f}$ and $\left.\tilde{g}=\tilde{f}-\frac{1}{2} f\right)$

$$
S=\frac{f^{\prime}(0)}{24}+S^{\prime}-\frac{1}{4} \int_{0}^{\infty} \frac{f(r)}{\operatorname{sh} \pi r} d r .
$$

Let $F(s)$ be the Mellin transform of $f$, then (see 3.761.4 of [Gr-Ry])

$$
\begin{aligned}
S & =\frac{1}{2 \pi i} \int_{\sigma_{1}-i \infty}^{\sigma_{1}+i \infty} F(s)\left(\eta_{1}(s)-\frac{1}{2} \eta_{2}(s)\right) d s \\
S^{\prime} & =\frac{1}{2 \pi i} \int_{\sigma_{2}-i \infty}^{\sigma_{2}+i \infty} F(s) \pi^{s-1} \Gamma(1-s) \cos \frac{\pi s}{2}\left(\eta_{1}(1-s)-\eta_{2}(1-s)\right) d s
\end{aligned}
$$

where $2<\sigma_{1}<3,-2<\sigma_{2}<-1$ and

$$
\eta_{1}(s)=\sum \frac{N^{*}(4 n)}{\sqrt{4 n}}(\sqrt{4 n})^{-s} \quad \eta_{2}(s)=\sum \frac{N^{*}(n)}{\sqrt{n}}(\sqrt{n})^{-s}
$$

In Th. 2 of [Sh] it is proved that $\eta_{1}, \eta_{2}$ have a meromorphic continuation and are related by a certain functional equation (note that $\xi_{-}^{*}(s)=\eta_{1}(2 s-$ $1), \xi_{-}(s)=\eta_{2}(2 s-1)$ with the notation used there). After some elementary manipulations one can summarize these results saying that $\eta_{1}-\frac{1}{2} \eta_{2}$ is entire, takes the value $-1 / 48$ at -1 and it holds

$$
\begin{aligned}
& \eta_{1}(s)-\frac{1}{2} \eta_{2}(s) \\
& =\pi^{s-1} \Gamma(1-s) \cos \frac{\pi s}{2}\left(\eta_{1}(1-s)-\eta_{2}(1-s)\right)+\frac{1}{4}\left(1-2^{1-s}\right) \zeta(s) \csc \frac{\pi s}{2}
\end{aligned}
$$

Hence, moving the line of integration in $S$ to $\operatorname{Re} s=\sigma_{2}$, it follows (note that $\left.\operatorname{Res}_{s=-1} F(s)=f^{\prime}(0)\right)$

$$
S=-\frac{f^{\prime}(0)}{48}+S^{\prime}+\frac{1}{8 \pi i} \int_{\sigma_{2}-i \infty}^{\sigma_{2}+i \infty} F(s)\left(1-2^{1-s}\right) \zeta(s) \csc \frac{\pi s}{2} d s
$$


Using again residue theorem and the functional equation of $\zeta$, we have

$$
\begin{aligned}
\frac{1}{8 \pi i} \int_{\sigma_{2}-i \infty}^{\sigma_{2}+i \infty} F(s)\left(1-2^{1-s}\right) \zeta(s) \csc \frac{\pi s}{2} d s \\
\quad=\frac{f^{\prime}(0)}{16}+\frac{1}{8 \pi i} \int_{\sigma_{3}-i \infty}^{\sigma_{3}+i \infty} F(s) C(1-s) d s
\end{aligned}
$$

where $-1<\sigma_{3}<0$ and $C(s)$ coincides with the Mellin transform of $-\operatorname{csch}(\pi x)$ (see 17.43.26 of [Gr-Ry]), hence (3.1) follows after substituting in (3.2) and applying Mellin convolution theorem (see 17.42.4 of [Gr-Ry]).

LEMMA 3.2. For $R>1$ and $0<\Delta<1$

$$
\Phi_{1}(R)=\frac{2 \pi}{9} R^{3}+\frac{\pi R^{2} \Delta}{3}-\frac{R^{2}}{2}+S_{1}+O\left(R^{1+\epsilon}\right)
$$

and

$$
\Phi_{2}(R)=\frac{\pi}{18} R^{3}+\frac{\pi R^{2} \Delta}{12}-\frac{R^{2}}{4}+S_{2}+O\left(R^{1+\epsilon}\right)
$$

where

$$
S_{1}=\frac{2 R}{\pi^{2} \Delta} \sum_{n} \frac{N(n)}{n^{3 / 2}} \sin (\pi \Delta \sqrt{n}) \cos (\pi(2 R+\Delta) \sqrt{n})
$$

and

$$
S_{2}=\frac{R}{2 \pi^{2} \Delta} \sum_{n} \frac{N(4 n)}{n^{3 / 2}} \sin (\pi \Delta \sqrt{n}) \cos (\pi(2 R+\Delta) \sqrt{n}) .
$$

Proof. If $g(x)=2 f_{R}(x / 2)$, we have

$$
\tilde{g}(x)=\frac{\sin (2 \pi R x)}{\pi^{2} x^{2}}-\frac{2 R \sin (\pi \Delta x) \cos (\pi(2 R+\Delta) x)}{\pi^{2} x^{2} \Delta} .
$$

Now we substitute in Lemma 3.1 (the lack of regularity of $g$ is less important because Lemma 4.1 assures the uniform convergence on compacta of the involved series). By 3.981.1 of [Gr-Ry]

$$
\int_{0}^{\infty} \frac{\tilde{g}(r)}{\operatorname{sh} \pi r} d r=\frac{1}{2} \int_{0}^{\infty} g(t) \operatorname{th}(\pi t / 2) d t=R^{2}+O\left(R^{1+\epsilon}\right)
$$

for any $\epsilon>0$, the rest of the calculations are plain getting

$$
\Phi_{1}(R)=\frac{2 \pi}{9} R^{3}+\frac{\pi R^{2} \Delta}{3}-\frac{R^{2}}{2}-\sum \frac{N^{*}(n)}{\sqrt{n}} \tilde{g}(\sqrt{n})+O\left(R^{1+\epsilon}\right) .
$$

Hence, using Lemma 4.1 to clear the right hand side, the result for $\Phi_{1}$ follows. The proof for $\Phi_{2}$ is similar but choosing $g(x)=2 \tilde{f}_{R}(x)$. 


\section{$\S 4$. Conclusion of the proof}

In this section we shall deduce Theorem 1.1 and Theorem 1.2 from the following lemmas which are proved appealing to the corresponding results of [Ch-Iw]. As usually, $\epsilon$ indicates a positive arbitrarily small real number and $n \asymp N$ means $c_{1} N<n<c_{2} N$ for some unspecified positive constants $c_{1}, c_{2}$.

LEMMA 4.1. If $R>1$ then

$$
\begin{aligned}
\sum_{n \asymp N} N(n) e(R \sqrt{n}) \ll N^{5 / 4+\epsilon}+ & N^{\epsilon} \min \left(R^{3 / 8} N^{15 / 16}\right. \\
& \left.+R^{1 / 8} N^{17 / 16}, R^{7 / 24} N^{49 / 48}+R^{5 / 24} N^{53 / 48}\right),
\end{aligned}
$$

and a similar result holds when $N(n)$ is replaced by $N(4 n)$.

LEMMA 4.2. With the notation of Lemma 2.2, if $R^{-1}<\Delta(R)<1$

$$
\Psi_{1}(R)=-\frac{\pi R^{2} \Delta}{4}+O\left(R^{15 / 8+\epsilon} \Delta^{7 / 8}+R^{83 / 48+\epsilon} \Delta^{2 / 3}\right)
$$

and

$$
\Psi_{2}(R)=-\frac{\pi R^{2} \Delta}{12}+O\left(R^{15 / 8+\epsilon} \Delta^{7 / 8}+R^{83 / 48+\epsilon} \Delta^{2 / 3}\right)
$$

Proof of Lemma 4.1. Let $S_{N}$ be the sum of the lemma, by Lemma 2.1 it can be written as a three-dimensional exponential sum and some manipulations using Lemma 7.3 of [Gr-Ko] prove (see the details in Lemma 3.1 of $[\mathrm{Ch}-\mathrm{Iw}])$

$$
\begin{aligned}
S_{N} & \ll N^{1+\epsilon}+\left|\sum_{x, y, z} e\left(R \sqrt{4 x z-y^{2}}\right)\right| \\
& \ll N^{1+\epsilon}+N^{\epsilon} \sum_{u \asymp N}\left|\sum_{y \ll N^{1 / 2}} e(\theta y) e\left(R \sqrt{u-y^{2}}\right)\right|
\end{aligned}
$$

for some $\theta \in \mathbb{R}$. Splitting the range of the inner sum into subintervals of lenght $N^{1 / 2-\epsilon}$, by Cauchy's inequality

$$
S_{N}^{2} \ll N^{1+\epsilon} \sum_{y_{1}, y_{2}}\left|\sum_{u \asymp N} e\left(R\left(\sqrt{u-y_{1}^{2}}-\sqrt{u-y_{2}^{2}}\right)\right)\right|
$$


with $1<y_{1} \leq y_{2}<y_{1}+N^{1 / 2-\epsilon}<N^{1 / 2}$, hence there exists $1 \leq D<N^{1-\epsilon}$ such that

$$
S_{N}^{2} \ll N^{5 / 2+\epsilon}+N^{1+\epsilon} \sum_{v \asymp D}\left|\sum_{u \asymp N} e(R(\sqrt{u}-\sqrt{u+v}))\right|
$$

and this sum was bounded in the proof of Lemma 3.1 of [Ch-Iw] getting the expected result. The proof when $N(n)$ is replaced by $N(4 n)$ is completely similar.

Proof of Lemma 4.2. Theorem 4.1 of [Ch-Iw] implies for $1<K<N^{1 / 2}$

$$
\sum_{N<n \leq N+K} L(1, \chi-4 n)=\frac{\pi^{2}}{7 \zeta(3)} K+O\left(K^{7 / 8} N^{\epsilon}+K^{2 / 3} N^{1 / 32+\epsilon}\right)
$$

and the same method proves

$$
\sum_{\substack{N<n \leq N+K \\ n \equiv 0,3(4)}} L\left(1, \chi_{-n}\right)=\frac{\pi^{2}}{12 \zeta(3)} K+O\left(K^{7 / 8} N^{\epsilon}+K^{2 / 3} N^{1 / 32+\epsilon}\right) .
$$

By class number formula and Abel's Lemma

$$
\Psi_{1}(R)=-\frac{R}{\pi \Delta} \sum_{2 \nmid l} \int_{R^{2} l^{-2}}^{(R+\Delta)^{2} l^{-2}} t^{-1 / 2} \sum_{R^{2} l^{-2}<n \leq t} L\left(1, \chi_{-4 n}\right) d t+O\left(R^{1+\epsilon}\right)
$$

and

$$
\Psi_{2}(R)=-\frac{R}{2 \pi \Delta} \sum_{l} \int_{R^{2} l^{-2}}^{(R+\Delta)^{2} l^{-2}} t^{-1 / 2} \sum_{R^{2} l^{-2}<n \leq t} L\left(1, \chi_{-n}\right) d t+O\left(R^{1+\epsilon}\right)
$$

then the result follows from (4.1) and (4.2).

Proof of Theorem 1.1 and Theorem 1.2. Dividing the range of summation of $S_{1}$ and $S_{2}$ in Lemma 3.2 according $n \ll \Delta^{-2}$ or $n \gg \Delta^{-2}$ and applying Lemma 4.1 in combination with the trivial bound, we conclude that for $0<\Delta<R^{-1 / 2}$

$$
\max \left(\left|S_{1}\right|,\left|S_{2}\right|\right) \ll\left(R \Delta^{-1 / 2}+R^{9 / 8} \Delta^{-1 / 8}+R^{21 / 16}\right) \Delta^{-\epsilon},
$$

and choosing $\Delta(R)=R^{-7 / 11}$ Theorem 1.1 and Theorem 1.2 follow from Lemma 2.2, Lemma 3.2 and Lemma 4.2 . 
NOTE ADDED IN PROOF. After this paper was accepted for publication, D. R. Heath-Brown has proved ("Lattice points in the sphere," to appear in the proceedings of the Number Theory Conference in honour of Prof. A. Schinzel held in Zakopane, Poland, 1997) that the error term in Lemma 4.2 can be improved to $\left(R^{11 / 6} \Delta^{5 / 6}+R^{7 / 6} \Delta^{-1 / 6}+R^{19 / 15}\right) R^{\epsilon}$. Inserting this bound in the final optimization and choosing $\Delta(R)=R^{-5 / 8}$, it is obtained that the 29/44 exponent in Theorem 1.1 and 1.2 can be replaced by $21 / 32$.

\section{REFERENCES}

[Ch] Chen, Jing-Run, Improvement on the asymptotic formulas for the number of lattice points in a region of the three dimensions (II), Sci. Sinica, 12 N. 6 (1963), 751-764.

[Ch-Iw] F. Chamizo and H. Iwaniec, On the sphere problem, Revista Mat. Iberoamericana, 11, N. 2 (1995), 417-429.

[Da] H. Davenport, Multiplicative Number Theory, Second edition, Graduate texts in Mathematics 74, Springer-Verlag, 1980.

[Di] P. G. L. Dirichlet, Vorlesungen über Zahlentheorie, (supplemented and revised by R. Dedekind), Braunschweig, 1863, (Reprinted by Chelsea Publishing Company, New York 1968).

[Ga] C. F. Gauss, Disquisitiones Arithmeticae, Leipzig, 1801, (English Translation by A. A. Clarke, Yale University Press, New Haven 1966).

[Gr-Ko] S. W. Graham and G. Kolesnik, Van der Corput's Method of Exponential Sums, London Math. Soc. Lecture Notes Series, 126, Cambridge University Press, 1991.

[Gr-Ry] I.S. Gradshteyn - I.M. Ryzhik., Table of Integrals, Series and Products, Fifth edition (edited by A. Jeffrey), Academic Press, 1994.

[Sh] T. Shintani, Zeta functions associated with the vector space of quadratic forms, J. Fac. Sci. Univ. Tokyo. Sect IA, 22 (1975), 22-65.

[Vi] I.M. Vinogradov, On the number of integer points in a sphere, Izv]. Akad. Nauk SSSR Ser. Mat., 27 (1963), 957-968, (Russian).

Fernando Chamizo

Departamento de Matemáticas

Facultad de Ciencias

Universidad Autónoma de Madrid

28049 Madrid, Spain

fernando.chamizo@uam.es

Henryk Iwaniec

Department of Mathematics

Rutgers University

New Brunswick, NJ 08903, U.S.A.

iwaniec@math . rutgers.edu 